Supporting Information For:

\title{
Enantiospecificity of Cysteine Adsorption on a Ferromagnetic Surface: Is it Kinetically or Thermodynamically Controlled?
}

\author{
Y. Lu, B. P. Bloom, S. Qian, and D. H. Waldeck* \\ Chemistry Department, University of Pittsburgh, Pittsburgh PA 15260 \\ Corresponding Author Email: dave@pitt.edu
}

(412) 624-8430

\section{EQCM system}

The EQCM experiments were performed using a 7.9995 MHz quartz crystal with an EQCM cell attachment and a 430A potentiostat (CH Instruments). The surface area of the crystal is $0.205 \mathrm{~cm}^{2}$ and is coated with $100 \mathrm{~nm}$ of nickel and $10 \mathrm{~nm}$ polycrystalline gold as the working electrode area (CH Instruments). The counter electrode was Pt wire and the reference electrode was a saturated $\mathrm{Ag} / \mathrm{AgCl}$ electrode. For measurements using a magnetic field, a $0.54 \mathrm{~T}$ magnet was placed underneath the cell; see Figure S1. Because of the decay in magnetic field with distance, and the fundamentally large distance between the surface of the electrode and the surface of the magnet, the use of a stronger magnet was desired.

\section{Underpotential deposition of $\mathbf{P b}$}

To ensure the quartz crystal was functioning appropriately, underpotential deposition (UPD) experiments using lead were performed using a solution comprising 5 $\mathrm{mM}$ of $\mathrm{Pb}\left(\mathrm{ClO}_{4}\right)_{2}, 10 \mathrm{mM}$ of $\mathrm{HClO}_{4}$, and $0.1 \mathrm{M}$ of $\mathrm{KClO}_{4}$ - see Figure S1. Cyclic voltammograms (left panel) from $0.24 \mathrm{~V}$ to $-0.45 \mathrm{~V}$ versus saturated $\mathrm{Ag} \mid \mathrm{AgCl}$ in tandem with the QCM showed that lead deposits and gives rise to a cathodic peak at $-0.31 \mathrm{~V}$ and an anodic peak at $-0.2 \mathrm{~V}$. The frequency change (right panel) was correlated to the mass of the UPD (63 ng) and corresponds to a coverage of $1.5 \times 10^{19} \mathrm{~mol} / \mathrm{cm}^{2}$, in agreement with other reports. ${ }^{1}$ The UPD measurements indicate that the EQCM is functioning accurately. 

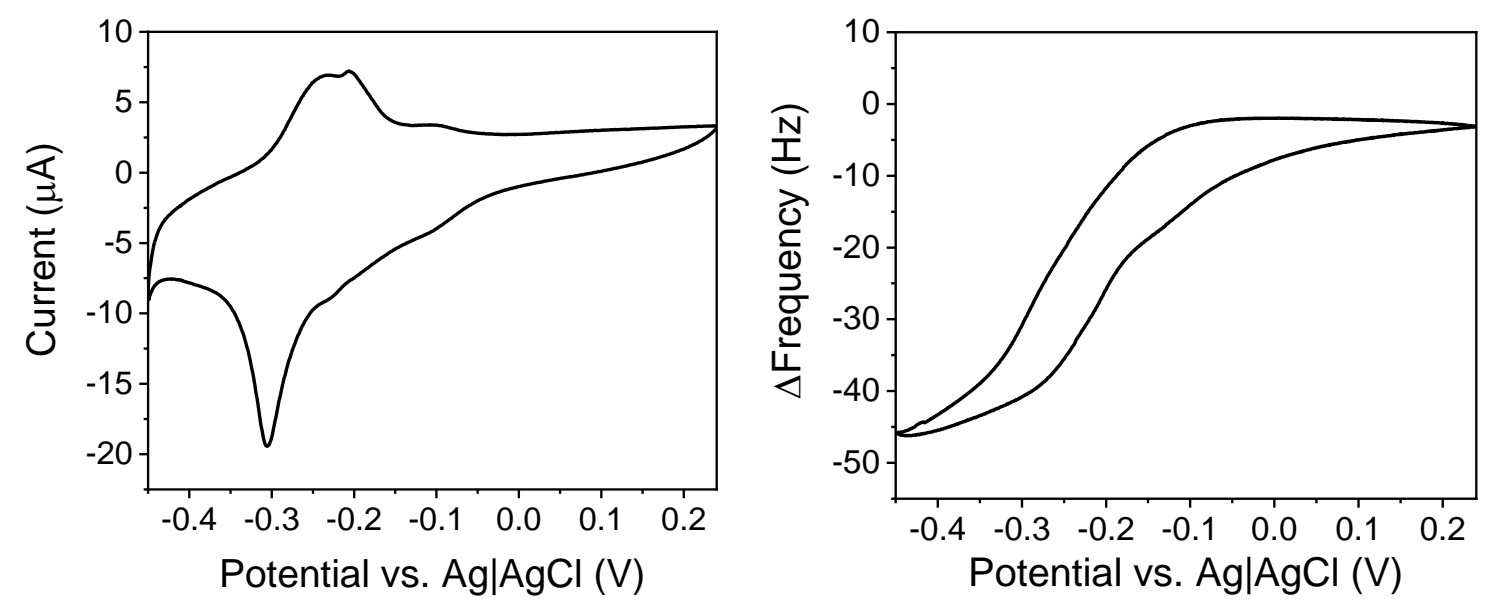

Figure S1. The voltametric (left) and frequency (right) response of the QCM during the deposition of $\mathrm{Pb}$.

\section{Adsorption Isotherm}

Cyclic voltammetry and QCM measurements were used to build the data for the adsorption isotherms. The data were collected by scanning from $-0.4 \mathrm{~V}$ to $-1.1 \mathrm{~V}$ versus saturated $\mathrm{Ag} \mid \mathrm{AgCl}$ at a scan rate $=25 \mathrm{mV} / \mathrm{s}$. Unless specified all measurements were made in a $\mathrm{pH} 8$ phosphate buffer solution. The system was allowed to equilibrate for one hour and the frequency change from the $30^{\text {th }}$ cycle, corresponding to the oxidative adsorption of cysteine on a gold substrate, was converted to a change in mass $(\Delta m)$ according to the Sauerbrey equation by a factor of $-1.4 \mathrm{ng} / \mathrm{Hz}$. The excessive amount of cycles was necessary to allow the instrument to reach equilibrium and give consistent results. Next, the mass change $(\Delta m)$ or concentration/ $\Delta m$ was plotted versus concentration and fit to a Langmuir - Freundlich isotherm model or a linear isotherm.

\section{Linearization of isotherm equation.}

Assuming that the adsorption is a reversible process

$$
A+S \rightleftharpoons A S
$$

where $A$ represent the adsorbate, $S$ represent unoccupied adsorbing sites on the adsorbent, and $A S$ represent occupied sites. The equilibrium constant is

$$
K_{a d s}=\frac{[A S]}{[A][S]}
$$

Note, here $[A]$ is the concentration of the adsorbate $A$ in the solution.

The fractional occupancy of the adsorbate can be calculated from the EQCM:

$$
\theta=\frac{\Delta m}{m_{\operatorname{Max}}}
$$


where $\Delta m$ is the mass of the adsorbate measured by the EQCM, and $\Delta m_{\operatorname{Max}}$ represents the mass for a monolayer coverage. Thus, the general form of the adsorption isotherm becomes:

$$
\Delta m=\frac{\Delta m_{\text {Max }} K_{a d s} c_{c y s}}{1+K_{a d s} c_{c y s}}
$$

where $c_{c y s}$ is equivalent to $[A]$ - the concentration of cysteine. Algebraic rearrangement of this expression gives the adsorption isotherm in a linear form as

$$
\frac{c_{c y s}}{\Delta m}=c_{c y s} \cdot \frac{1}{\Delta m_{\max }}+\frac{1}{K_{a d s} \cdot \Delta m_{\max }}
$$

Thus, the maximum adsorption amount and the adsorption equilibrium constant $K_{a d s}$ can be calculated by the slope and intercept value of the linear plot. From the $K_{a d s}$, the Gibbs free energy of the adsorption can be calculated by:

$$
K_{\text {ads }}=\frac{1}{c_{\text {solvent }}} \cdot \exp \left(-\frac{\Delta_{a d s} G}{R T}\right)
$$

where $c_{\text {solvent }}$ is the concentration of the solvent, which is usually close to the concentration of pure solvent for low concentration solutions. Figure S2 shows a standard (left) and linearized (right) Langmuir isotherm for D-cysteine. The average $\Delta_{\text {ads }} G$ was found to be $-34.3 \pm 1.2 \mathrm{~kJ} / \mathrm{mol},-34.0 \pm 1.0 \mathrm{~kJ} / \mathrm{mol}$ and $-34.2 \pm 1.5 \mathrm{~kJ} / \mathrm{mol}$ in three separate trials.
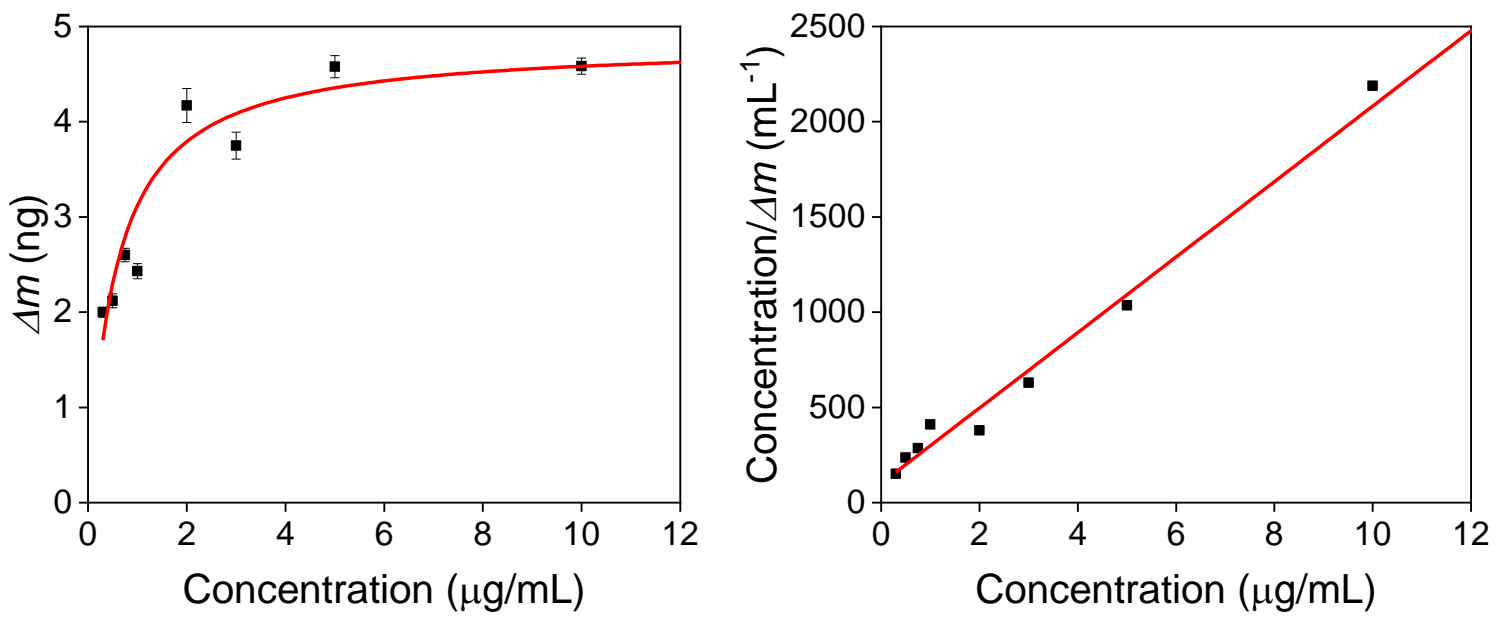

Figure S2. The left panel shows an adsorption isotherm of D-cysteine using the change in mass determined from the cyclic voltammetry measurements at different cysteine concentrations. The red line is a Langmuir isotherm fit to the data. The right panel shows a linearized Langmuir isotherm plot of the same data in left panel.

\section{Kinetic Experiments}


The quartz crystal was first incubated in the cysteine solution for $1 \mathrm{~h}$ to allow the system to equilibrate before applying a potential. Cyclic voltammograms (CVs) were recorded for 30 cycles (condition: - $0.4 \mathrm{~V} \sim-1.1 \mathrm{~V}$ versus saturated $\mathrm{Ag} \mid \mathrm{AgCl}$, scan rate = $25 \mathrm{mV} / \mathrm{s}$ ), as well as the frequency response of the quartz crystal, to ensure the surface was stable and the desorption and adsorption process was reversible. The chronoamperometry experiments were performed immediately after the $\mathrm{CV}$ acquisition with an initial potential of $-0.8 \mathrm{~V}$ corresponding to reductive desorption of cysteine and then a more positive potential, $-0.5 \mathrm{~V}$, corresponding to oxidative adsorption. A $5 \mathrm{~s}$ pulse width was applied during the experiment and $>150$ cycles was performed to build up the statistics for the histogram plots. Unless specified, all measurements were made in a $\mathrm{pH} 8$ phosphate buffer solution. The experiments with cysteine methyl ester used the same general procedure as cysteine, however in some instances additional time was required for the system to equilibrate. For these studies, an additional $30 \mathrm{CV}$ cycles were collected.

\section{Kinetic model derivation}

Assuming a simple Langmuir model, the desorption rate can be written as

$$
r_{d e s}=-\frac{d \theta}{d t}=k_{d e s} \cdot \theta \text { or } \theta(t)=\theta_{t=0} \cdot \exp \left(-k_{d e s} \cdot t\right)
$$

where $\theta$ is the fraction of adsorbed cysteine on the surface (filled surface sites or coverage) and $k_{d e s}$ is the desorption rate constant. Thus, the rate constant extracted from the exponential fits for the desorption data correspond to $k_{d e s}$. For the case of adsorption, we can write the rate as

$$
r_{a d s}=\frac{d \theta}{d t}=k_{a d s} \cdot c_{c y s} \cdot(1-\theta)=k_{a d s}^{\prime} \cdot(1-\theta)
$$

where $c_{c y s}$ is the concentration of cysteine in solution, $k_{a d s}$ is the adsorption rate constant and $k^{\prime}{ }_{a d s}$ is the effective adsorption rate constant. To quantify the rates for the desorption and adsorption processes, the time responses of the QCM frequency were fit to an exponential growth equation $y=A \cdot e^{t / \tau_{1}}+y_{0}$ for desorption and an exponential decay equation $y=A \cdot e^{-t / \tau_{2}}+y_{0}$ for adsorption, where $A, y_{0}$, and $\tau_{1}$ and $\tau_{2}$ were adjusted for a best fit to the data. Then the desorption rate constant was calculated as $k_{d e s}=\frac{1}{\left|\tau_{1}\right|}$, and the effective adsorption rate constant as $k^{\prime}{ }_{a d s}=\frac{1}{\left|\tau_{2}\right|}$.

Note that this analysis makes the approximation that the adsorption and desorption rate processes are irreversible at the potentials $-0.5 \mathrm{~V}$ and $-0.8 \mathrm{~V}$ used in the experiments. A more elaborate analysis is possible for studies in which the back reaction is included. See the discussion of the 'temperature jump' method in reference 2.

\section{Histogram of different concentrations}

Figure S3 shows a histogram of $k^{\prime}$ ads for D-cysteine (left) and L-cysteine (right) at different concentrations under a North (red) or South (blue) magnetic field. A summary of 
the rate data are shown in Table S1. These data were used to construct the polarization plot shown in the main text in Figure 3. Table $\mathrm{S} 2$ and $\mathrm{S} 3$ summarize the $\mathrm{pH}$ dependent rate data for L-cysteine and L-cysteine methyl ester.
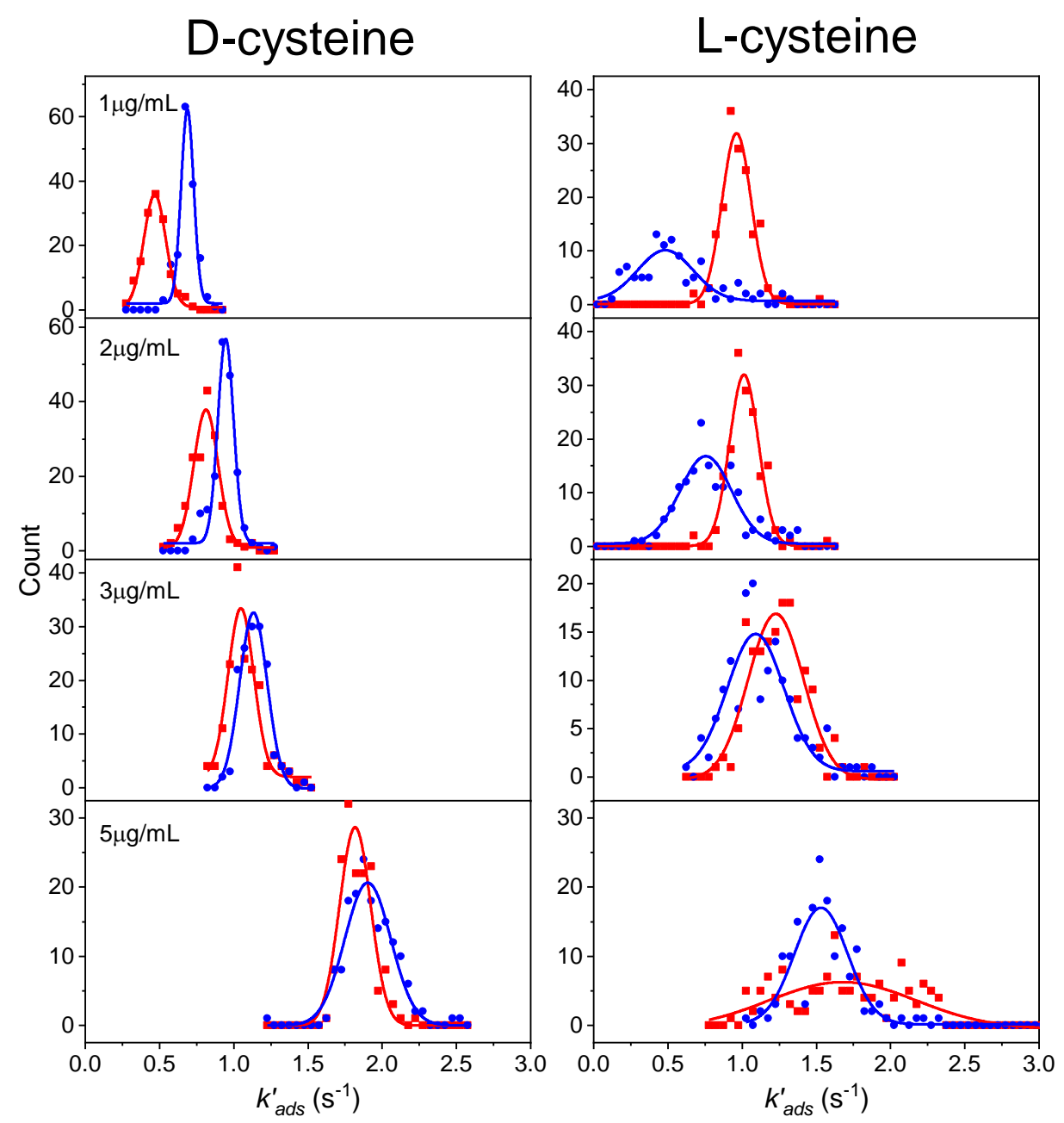

Figure S3. The histogram of the adsorption rate of D-cysteine (left) and L-cysteine (right) under a North magnetic field (red) and South magnetic field (blue) at different concentrations. A best fit of the data using a Gaussian distribution is shown as a solid line. 

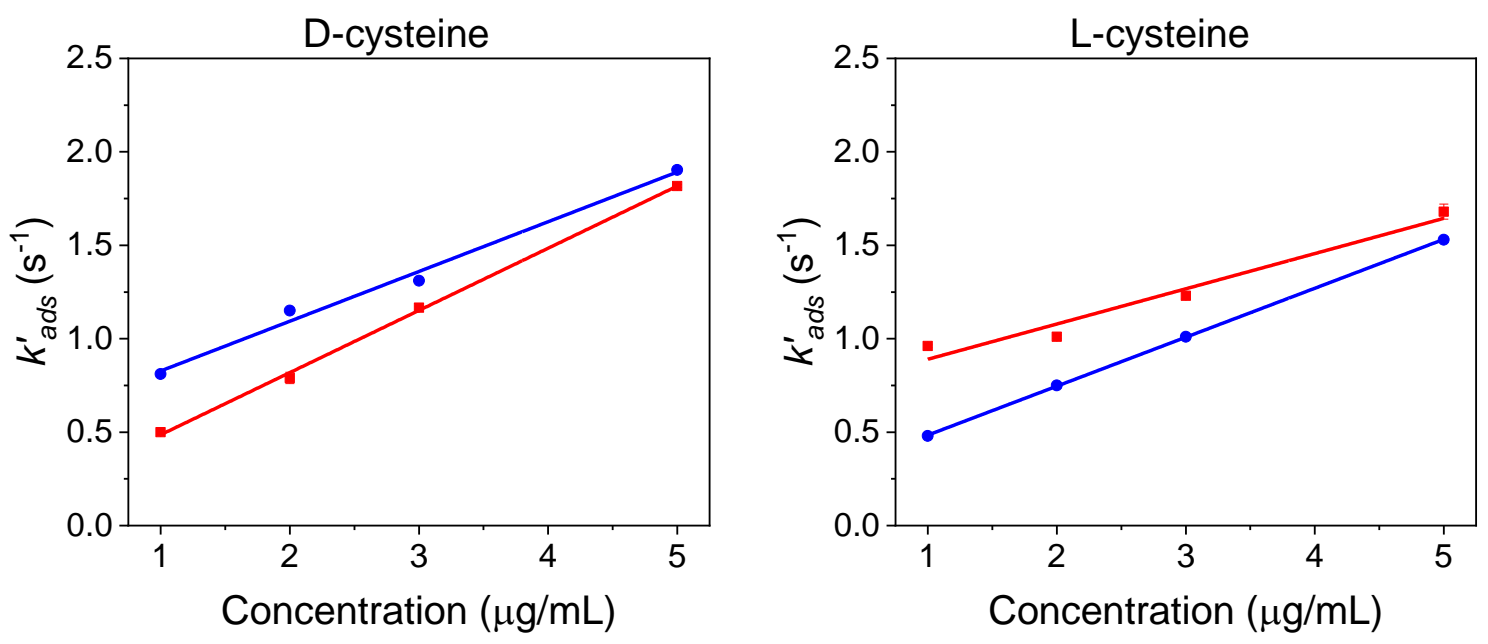

Figure S4. The adsorption rate $k_{a d s}$ is plotted as a function of the solution concentration for D-cysteine (left) and L-cysteine (right) under North magnetic field (red) and South magnetic field (blue). The error bars show the standard deviation of the mean of the effective rate.

Figure $\mathrm{S} 4$ shows that there is a linear relationship between $k^{\prime}$ ads and concentration for both D-cysteine (left panel) and L-cysteine (right panel) under North (red) and South (blue) magnetic field. For D-cysteine under a North (South) magnetic field $k_{\text {ads }}$ was 0.33

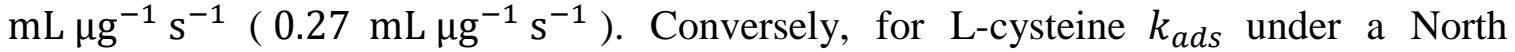
magnetic field and South magnetic field is $0.19 \mathrm{~mL} \mathrm{\mu g}^{-1} \mathrm{~s}^{-1}$ and $0.26 \mathrm{~mL} \mathrm{\mu g}^{-1} \mathrm{~s}^{-1}$, respectively.

Table S1. Summary of D-cysteine and L-cysteine adsorption rate constant and polarization at $\mathrm{pH} 8$

\begin{tabular}{|c|c|c|c|c|}
\hline & \multicolumn{4}{|c|}{ D-cysteine } \\
\hline $\begin{array}{c}\text { Conc. } \\
(\mu \mathrm{g} / \mathrm{mL})\end{array}$ & $\begin{array}{c}k_{\text {ads }}^{\prime} \mathrm{N} \\
\left(\mathrm{s}^{-1}\right)\end{array}$ & $\begin{array}{c}k_{\text {ads }}^{\prime} \mathrm{S} \\
\left(\mathrm{s}^{-1}\right)\end{array}$ & $P$ & $\sigma_{\bar{x}}$ \\
\hline 1 & 0.50 & 0.81 & $23.7 \%$ & $0.65 \%$ \\
\hline 2 & 0.79 & 1.15 & $18.5 \%$ & $1.64 \%$ \\
\hline 3 & 1.17 & 1.31 & $-5.8 \%$ & $0.36 \%$ \\
\hline 5 & 1.82 & 1.90 & $-2.3 \%$ & $0.22 \%$ \\
\hline \multicolumn{5}{|c|}{$\mathrm{L}-\mathrm{Cysteine}$} \\
\hline $\begin{array}{c}\text { Conc. } \\
(\mu \mathrm{g} / \mathrm{mL})\end{array}$ & $\begin{array}{c}k_{\text {ads }}^{\prime} \mathrm{N} \\
\left(\mathrm{s}^{-1}\right)\end{array}$ & $\begin{array}{c}k_{\text {ads }}^{\prime} \mathrm{S} \\
\left(\mathrm{s}^{-1}\right)\end{array}$ & $P$ & $\sigma_{\bar{x}}$ \\
\hline 1 & 0.96 & 0.48 & $33.3 \%$ & $0.56 \%$ \\
\hline 2 & 1.01 & 0.75 & $14.8 \%$ & $0.40 \%$ \\
\hline 3 & 1.23 & 1.01 & $9.8 \%$ & $0.62 \%$ \\
\hline 5 & 1.68 & 1.53 & $4.5 \%$ & $1.17 \%$ \\
\hline
\end{tabular}


Table S2. Summary of $1 \mu \mathrm{g} / \mathrm{mL}$ L-cysteine adsorption rate constant and polarization at different $\mathbf{p H}$

\begin{tabular}{|c|c|c|c|c|}
\hline $\mathbf{p H}$ & $\begin{array}{c}k_{a d s}^{\prime} \mathrm{N} \\
\left(\mathrm{s}^{-1}\right)\end{array}$ & $\begin{array}{c}k_{a d s}^{\prime} \mathrm{S} \\
\left(\mathrm{s}^{-1}\right)\end{array}$ & $P$ & $\sigma_{\bar{x}}$ \\
\hline $\mathbf{8}$ & 0.96 & 0.48 & $33.3 \%$ & $0.56 \%$ \\
\hline $\mathbf{8 . 1 1}$ & 0.941 & 0.727 & $12.8 \%$ & $0.50 \%$ \\
\hline $\mathbf{8 . 3 1}$ & 0.96 & 0.85 & $6.1 \%$ & $0.38 \%$ \\
\hline $\mathbf{8 . 4 6}$ & 0.67 & 0.77 & $-6.9 \%$ & $0.68 \%$ \\
\hline $\mathbf{8 . 5 6}$ & 0.718 & 0.963 & $-14.6 \%$ & $0.31 \%$ \\
\hline $\mathbf{9}$ & 1.36 & 1.55 & $-6.5 \%$ & $0.30 \%$ \\
\hline $\mathbf{1 0}$ & 1.07 & 1.2 & $-5.7 \%$ & $0.19 \%$ \\
\hline $\mathbf{1 1}$ & 1.01 & 1.05 & $-1.9 \%$ & $0.37 \%$ \\
\hline $\mathbf{1 2}$ & 0.656 & 0.665 & $-0.7 \%$ & $0.19 \%$ \\
\hline
\end{tabular}

Table S3. Summary of $1 \mu \mathrm{g} / \mathrm{mL}$ L-cysteine methyl ester adsorption rate constant and polarization at different $\mathrm{pH}$

\begin{tabular}{|c|c|c|c|c|}
\hline $\mathbf{p H}$ & $\begin{array}{c}k_{\text {ads }}^{\prime} \mathrm{N} \\
\left(\mathrm{s}^{-1}\right)\end{array}$ & $\begin{array}{c}k_{\text {ads }}^{\prime} \mathrm{S} \\
\left(\mathrm{s}^{-1}\right)\end{array}$ & $P$ & $\sigma_{\bar{x}}$ \\
\hline $\mathbf{6 . 6}$ & 1.61 & 2.2 & $-15.5 \%$ & $0.43 \%$ \\
\hline $\mathbf{6 . 8}$ & 1.9 & 1.86 & $1.1 \%$ & $0.62 \%$ \\
\hline $\mathbf{7}$ & 1.1 & 0.97 & $6.4 \%$ & $0.80 \%$ \\
\hline $\mathbf{8}$ & 1.07 & 0.93 & $6.9 \%$ & $0.58 \%$ \\
\hline $\mathbf{1 0}$ & 0.53 & 0.53 & $0 \%$ & $0.37 \%$ \\
\hline
\end{tabular}

\footnotetext{
${ }^{1}$ Deakin, M. R.; Melroy, O. Underpotential metal deposition on gold, monitored in situ with a quartz microbalance. J. Electroanal. Chem. Interf. Electrochem. 1988, 239, 321-331.

${ }^{2}$ H. Kuhn, H. D. Foersterling, and D. H. Waldeck Principles of Physical Chemistry, $2^{\text {nd }}$ ed. (Wiley, 2009, NY)
} 